\title{
Encefalite e encefalopatia em pacientes acometidos pela COVID-19
}

\author{
Encephalitis and encephalopathy in patients affected by COVID-19 \\ Encefalitis y encefalopatía en pacientes afectados por COVID-19
}

Daniele Alcoforado Costa

ORCID: https://orcid.org/0000-0001-8389-1248

Universidade Federal do Delta do Parnaíba, Brasil

E-mail: dani.a.costa712@gmail.com

Brenda Nascimento Dias

ORCID: https://orcid.org/0000-0002-9226-2133

Universidade Federal do Delta do Parnaíba, Brasil

E-mail: phbbrenda@gmail.com

Bruna Maria de Carvalho Pereira

ORCID: https://orcid.org/0000-0003-4527-3697

Universidade Federal do Delta do Parnaíba, Brasil

E-mail:brunaluna.bc@gmail.com

Francisco Alex da Rocha Coelho

ORCID: https://orcid.org/0000-0002-3308-375X

Universidade Federal do Delta do Parnaíba, Brasil

E-mail: alex123rocha@hotmail.com

Grazielle Araújo dos Santos

ORCID: https://orcid.org/0000-0002-3212-5153

Universidade Federal do Delta do Parnaíba, Brasil E-mail:graziellearj@gmail.com

Joana Darc Viana Silva

ORCID: https://orcid.org/0000-0003-0698-9334

Universidade Federal do Delta do Parnaíba, Brasil

E-mail: joanadarc.vs@hotmail.com

Maria Rikelly Frota Aguiar

ORCID: https://orcid.org/0000-0001-5041-8753

Universidade Federal do Delta do Parnaíba, Brasil E-mail: mariarikellyurca@ hotmail.com

Thayaná Fernandes

ORCID: https://orcid.org/0000-0002-6590-4620

Universidade Federal do Delta do Parnaíba, Brasil

E-mail: thayana.fernandes@ hotmail.com

Eduardo de Melo Prado

ORCID: https://orcid.org/0000-0002-5366-4316 Centro Universitário UNINTA, Brasil

E-mail: edumeloprado94@gmail.com

Rubens Renato de Sousa Carmo

ORCID: https://orcid.org/0000-0001-7135-7531

Universidade Federal do Delta do Parnaíba, Brasil

E-mail: rubensrenatobezerra@gmail.com

Antônio Thomaz Oliveira

ORCID: https://orcid.org/0000-0003-0277-6833

Universidade Federal do Delta do Parnaíba, Brasil

E-mail: thomaz159@gmail.com

\section{Resumo}

Além do trato respiratório ser o principal acometido pela COVID-19, o envolvimento neurológico não é incomum em pacientes internados podendo resultar em complicações graves. Encefalopatia e encefalite são complicações graves e devastadoras do Sistema Nervoso Central (SNC) que vêm sendo associadas à COVID-19. Esta revisão sistemática reúne dados existentes na literatura a respeito do desenvolvimento de diversos tipos de encefalites e encefalopatias em pacientes com COVID-19. Foi realizada busca nas bases de dados PubMed, Scopus e Web of Science, resultando em 231 artigos. Restaram 25 trabalhos para compor esta revisão sistemática. Entre eles, 2 foram estudos retrospectivos e 23 são relatos de casos. 12 estudos apresentaram casos com dados consistentes com encefalite e 14 com encefalopatias. Entre eles, alguns casos com tipos mais específicos foram observados mais de uma vez em nossas pesquisas: 3 de meningoencefalite, 3 de Acute Necrotizing Encephalopathy (ANE), 2 de Posterior Reversible Encephalopathy Syndrome (PRES), 2 de Acute Disseminated Encephalomyelitis (ADEM). Foram comuns os achados de hiperintensidades da substância branca, cortical ou subcortical. Hipodensidades e hipoatenuação de estruturas profundas da substância branca e hipodensidades focais das estruturas profundas, assim como múltiplas lesões envolvendo hipocampo, gânglios 
de base, medula, tronco encefálico, tálamo, dentre outras regiões. Estudos pós-morte já demonstraram a presença de partículas virais de SARS-CoV-2 em tecido cerebral. Esse envolvimento do SNC associado à COVID-19 é considerado indicador de prognóstico ruim, podendo levar a rápida deterioração clínica dos pacientes.

Palavras-chave: Neuropatia; SARS-CoV-2; Sistema Nervoso Central.

\begin{abstract}
Not only the respiratory tract being the main one affected by COVID-19, but also neurological involvement is not uncommon in hospitalized patients and can result in serious complications. Encephalopathy and encephalitis are serious and devastating complications of the Central Nervous System (CNS) that have been associated with COVID-19. This systematic review gathers existing data in the literature regarding the development of different types of encephalitis and encephalopathies in patients with COVID-19. A search was performed in the PubMed, Scopus and Web of Science databases, resulting in 231 articles. There were 25 works left to compose this systematic review. Among them, 2 were retrospective studies and 23 are case reports. 12 studies presented cases with data consistent with encephalitis and 14 with encephalopathies. Among them, some cases with more specific types were observed more than once in our research: 3 meningoencephalitis, 3 Acute Necrotizing Encephalopathy (ANE), 2 Posterior Reversible Encephalopathy Syndrome (PRES), 2 Acute Disseminated Encephalomyelitis (ADEM). Findings of white matter, cortical or subcortical hyperintensities were common. Hypodensity and hypoattenuation of deep white matter structures and focal hypodensity of deep structures, as well as multiple lesions involving hippocampus, basal ganglia, medulla, brainstem, thalamus, among other regions. Postmortem studies have already demonstrated the presence of SARS-CoV-2 viral particles in brain tissue. This CNS involvement associated with COVID-19 is considered an indicator of poor prognosis, which can lead to rapid clinical deterioration of patients.
\end{abstract}

Keywords: Neuropathy; SARS-CoV-2; Central Nervous System.

\title{
Resumen
}

Además de que el tracto respiratorio es el principal afectado por el COVID-19, la afectación neurológica no es infrecuente en los pacientes hospitalizados y puede derivar en complicaciones graves. La encefalopatía y la encefalitis son complicaciones graves y devastadoras del sistema nervioso central (SNC) que se han asociado con COVID-19. Esta revisión sistemática recopila datos existentes en la literatura sobre el desarrollo de diferentes tipos de encefalitis y encefalopatías en pacientes con COVID-19. Se realizó una búsqueda en las bases de datos PubMed, Scopus y Web of Science, dando como resultado 231 artículos. Quedaban 25 trabajos para componer esta revisión sistemática. Entre ellos, 2 eran estudios retrospectivos y 23 son informes de casos. 12 estudios presentaron casos con datos compatibles con encefalitis y 14 con encefalopatías. Entre ellos, algunos casos con tipos más específicos se observaron más de una vez en nuestra investigación: 3 meningoencefalitis, 3 encefalopatía necrotizante aguda (ANE), 2 síndrome de encefalopatía posterior reversible (SEPR), 2 encefalomielitis aguda diseminada (ADEM). Fueron frecuentes los hallazgos de hiperintensidades de la sustancia blanca, corticales o subcorticales. Hipodensidad e hipoatenuación de estructuras de sustancia blanca profunda e hipodensidad focal de estructuras profundas, así como múltiples lesiones que involucran hipocampo, ganglios basales, médula, tronco encefálico, tálamo, entre otras regiones. Los estudios post mortem ya han demostrado la presencia de partículas virales del SARS-CoV-2 en el tejido cerebral. Esta afectación del SNC asociada con COVID-19 se considera un indicador de mal pronóstico, que puede conducir a un rápido deterioro clínico de los pacientes.

Palabras clave: Neuropatía; SARS-CoV-2; Sistema Nervioso Central.

\section{Introdução}

O Coronavírus da Síndrome Respiratória Aguda Grave 2 (SARS-CoV-2) foi detectado incialmente em pacientes com pneumonia de etiologia desconhecida em Wuhan, na China, em dezembro de 2019. Rapidamente o vírus se espalhou pelo mundo (Sheraton et al., 2020). Em março de 2020, a Coronavirus Disease (COVID-19) foi declarada como pandemia pela Organização Mundial de Saúde (WHO) (Kotfis et al., 2020). O trato respiratório é o principal acometido e mais estudado no contexto fisiopatológico da COVID-19. Entretanto, a evolução clínica da doença tem mostrado envolvimento sistêmico, afetando múltiplos órgãos e comprometendo as funções dos sistemas digestivo, cardiovascular e nervoso. Assim, o envolvimento neurológico não é incomum em pacientes internados e pode resultar em complicações graves se não for detectado e gerenciado precocemente (Ahmad \& Rathore, 2020).

Romero-Sánchez et al., 2020 descreveram em um estudo com mais de 800 pacientes que metade apresentou algum tipo de alteração neurológica, principalmente em pacientes com quadro grave da doença (Ahmad \& Rathore, 2020) Além de sintomas neurológicos mais frequentes como cefaleia, náusea e vômitos (Moriguch et al., 2020) ou hiposmia, anosmia e disgeusia (Bonzano et al., 2020), a infecção pode levar a complicações mais graves como distúrbios da consciência, doença cerebrovascular, encefalite e encefalopatia (Bridwell et al., 2020). Também já foi relatada influência da COVID-19 na progressão de estados de ansiedade e depressão (Li et al., 2020).

Encefalopatia e encefalite são complicações graves do Sistema Nervoso Central (SNC) associadas ao SARS-CoV-2 (Garg et al., 
2020). É possível que a invasão do SNC pelo SARS-CoV-2 seja um dos mecanismos sistêmicos que levam o paciente com COVID-19 ao óbito (Tassorelli et al., 2020). Portanto, é importante que se conheça a respeito dos mecanismos e consequências do envolvimento neurológico nos pacientes (Romero-Sánchez et al., 2020). Esta revisão se propõe a reunir os dados existentes na literatura a respeito do desenvolvimento de diversos tipos de encefalites e encefalopatias em pacientes com a COVID-19.

O SARS-CoV-2 apresenta manifestações clínicas semelhantes a outros coronavírus (CoVs) também causadores de doenças respiratórias graves, como o da Síndrome Respiratória do Oriente Médio (MERS) e o da a Síndrome Respiratória Aguda Grave (SARS) em humanos (Sepehrinezhad et al., 2020). Além disso, já se sabe que alguns CoVs humanos têm a capacidade de neuroinvasão (Sepehrinezhad et al., 2020; Carod-Artal, 2020) e da mesma forma que estes, o SARS-CoV parece ter uma atividade neuroinvasiva e neurotrópica (Bonzano et al., 2020).

Muito do que se conhece sobre a fisiopatologia do SARS-CoV-2 foi a partir de estudos retrospectivos sobre o vírus SARS-CoV (2002-2004). Ambos são betacoronavírus da linhagem B e demonstram grandes similaridades. Os dois CoVs utilizam como porta de entrada a Enzima Conversora da Angiotensina 2 (ECA-2) presente em diversos tecidos do corpo, predominantemente no epitélio dos alvéolos pulmonares, mas também presente em outras estruturas como mucosa oral e nasal, nasofaringe, intestino delgado, cólon, gânglios linfáticos, timo, medula óssea, baço, fígado, rim e cérebro (Danser et al., 2020; Klok et al., 2020).

Pelo seu potencial neurotropismo, o SARS-CoV também pode induzir encefalite e já foi encontrado no líquido cefalorraquidiano (LCR) (Hung et al., 2003) e no parênquima cerebral (Xu et al., 2005). Já foi também demonstrado em roedores que esse vírus possui tropismo pelo tecido cerebral do tálamo e do rombencéfalo, sugerindo que uma rota trans-sináptica entre os dendritos pode ser usada pelo vírus para a disseminação ( $\mathrm{Li}$ et al., 2020). Contudo, o SARS-CoV-2 mostra um potencial infeccioso maior que o primeiro, uma vez que se liga mais fortemente ao receptor ECA-2 (Li et al., 2020; Lu et al., 2020).

Embora o mecanismo exato pelo qual a SARS-CoV-2 cause encefalite/encefalopatias seja ainda desconhecido, acredita-se que ele possa seguir por várias rotas, incluindo transferência trans-sináptica através de neurônios infectados do endotélio vascular ou da barreira hematoencefálica. Existem dois mecanismos possíveis para a invasão da barreira: o primeiro é através da infecção e transporte pelas células endoteliais vasculares, que expressam ECA-2; o segundo é através da infecção de leucócitos que chegam ao SNC (Zubair et al., 2020). O vírus pode ainda infectar as células do sistema imunológico no SNC como macrófagos, micróglia e astrócitos (Condie, 2020). A invasão também pode se dar através do sistema nervoso entérico após a infecção do trato gastrointestinal, além do acesso por meio dos nervos vagos aferentes do pulmão (Toljan, 2020). A partir desses diversos meios o SARS-CoV-2 atinge o SNC, podendo resultar no desenvolvimento de algum tipo de encefalite ou encefalopatia, tanto por conta de danos virais diretos quanto pela tempestade de citocinas associada à infecção (Toljan, 2020; Mehta, 2020).

A ECA-2 é expressa nas células do tronco cerebral, sendo nessa área que ocorre a regulação das atividades cardiorrespiratórias e do sistema motor, sugerindo que o ataque ocasionado pelo SARS-CoV-2 nessas células possa também ser responsável pela disfunção respiratória, além da lesão pulmonar direta (Werner et al., 2020). Ademais, a invasão viral causa um processo inflamatório sistêmico, havendo o comprometimento da barreira hematoencefálica e uma consequente perturbação da homeostase do tecido cerebral. Tal desequilíbrio compromete muitas atividades sensoriais, afetando também a regulação respiratória e cardiovascular (Steardo et al., 2020). Outros fatores também agravam os sintomas neurológicos, como a hipóxia decorrente da hipoventilação pulmonar. O resultado dessa série de problemas é o que determina os efeitos neuropsiquiátricos do novo coronavírus (Steardo, 2020; Netland, 2008).

\section{Metodologia}

O presente estudo trata-se de uma revisão sistemática da literatura de caráter qualitativo. As revisões sistemáticas são de grande valia para o entendimento e interpretação de achados científicos envolvendo a compreensão dos níveis de evidência disponíveis. Nesse tipo de desenho de trabalho, é realizada uma compilação e análise de conjuntos de dados de estudos encontrados a partir de buscas na literatura, 
seguindo um rígido protocolo para reduzir a ocorrência de vieses e eliminar estudos de pouca relevância e de baixa qualidade (LinaresEspinós et al. 2018).

Para a escrita deste trabalho, foi seguido o protocolo geral de uma revisão sistemática descrito por Linares-Espinós et al. (2018). Foram acessadas as bases de dados PubMed, Scopus e Web of Science e, para a busca, utilizados os descritores específicos: "encephalitis”, “encephalopathy”, “COVID-19” e "SARS-CoV-2, combinados entre si por meio de descritores booleanos, 1 AND e 2 OR. Para a construção da revisão sistemática foi utilizado o protocolo Preferred Reporting Items for Systematic Reviews and Meta-Analysis (PRISMA).

Como critérios de inclusão: artigos completos, sem restrição de idioma, publicados no recorte temporal de dezembro de 2019 a junho de 2020. Foram considerados apenas os estudos que comprovaram a infecção pelo SARS-CoV-2 pelo método diagnóstico de Reverse Transcription Polymerase Chain Reaction (RT-PCR) e com quadro clínico-laboratorial de encefalite e/ou encefalopatia.

Ao final da busca, 231 artigos foram encontrados. Destes, 150 no PubMed, 56 na base de dados Scopus e 25 no Web of Science. A priori, foram excluídos 57 artigos por repetição. Dos 174 restantes, 149 trabalhos não atendiam aos critérios de inclusão e também foram excluídos, restando, portanto, 25 trabalhos para compor esta revisão. Dos estudos selecionados, 2 foram estudos retrospectivos e 23 são relatos de casos (Figura 1).

Figura 1: fluxograma para identificação, triagem, elegibilidade e análise dos estudos incluídos na revisão de literatura.

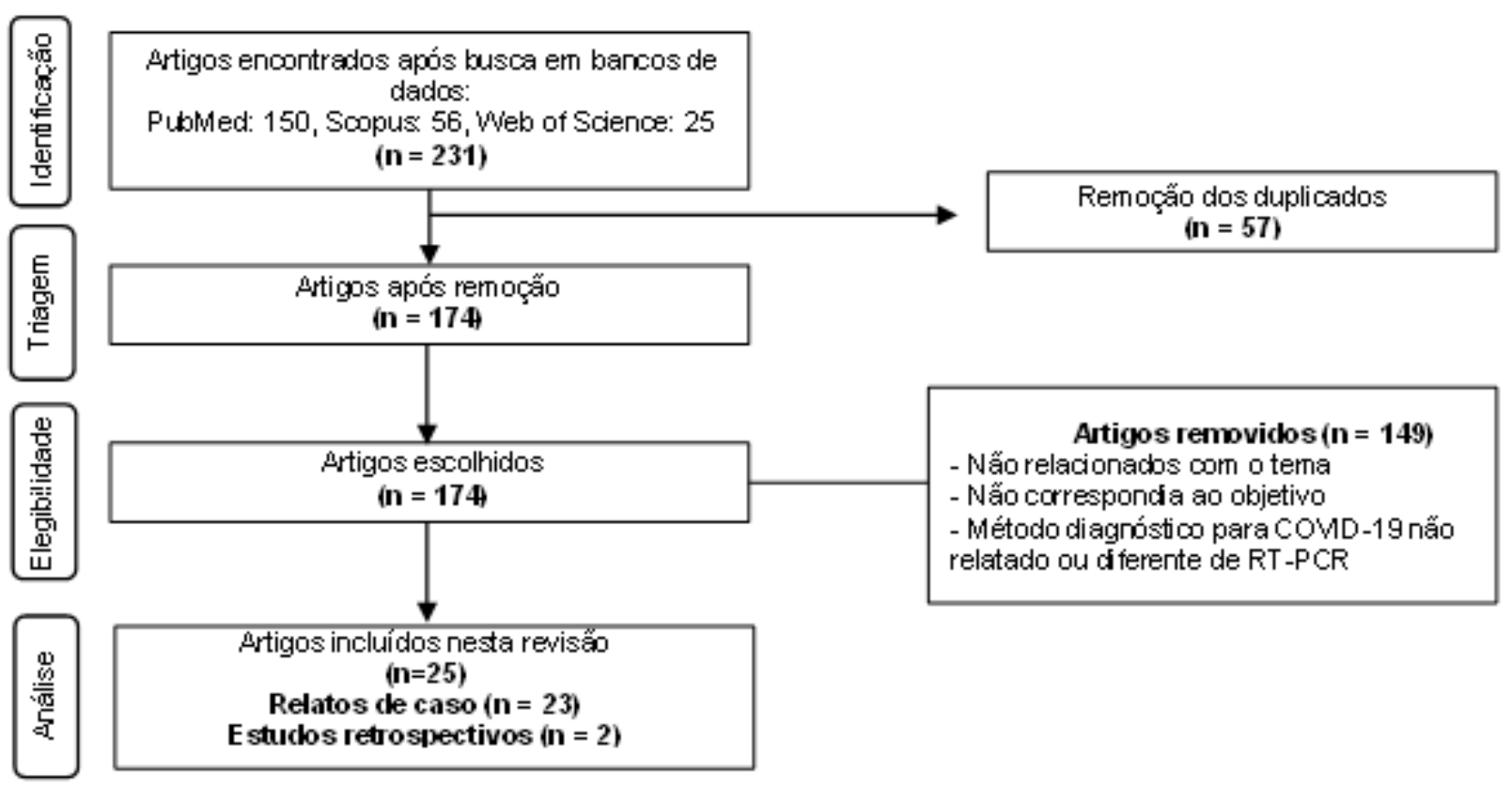

Fonte: Autores.

\section{Resultados}

As publicações selecionadas encontram-se compiladas e discorridas no Quadro 1, com a descrição de autores e ano de publicação, tipos de estudo, amostragem utilizada e os principais resultados de cada trabalho. 
Quadro 1: Trabalhos selecionados.

\begin{tabular}{|c|c|c|c|}
\hline Autores e ano & Tipo de estudo & Amostragem & Principais resultados \\
\hline $\begin{array}{l}\text { (Scullen et al., } \\
\text { 2020) }\end{array}$ & $\begin{array}{l}\text { Transversal } \\
\text { retrospectivo }\end{array}$ & $\begin{array}{l}76 \text { pacientes admitidos } \\
\text { centro acadêmico } \\
\text { atendimento terciário em } \\
\text { Orleans, Louisiana, em estado } \\
\text { crítico e confirmado para } \\
\text { COVID-19. }\end{array}$ & $\begin{array}{l}\text { EEG alterada em } 44 \% \text { dos pacientes afetados, com oscilações de frequência delta e teta, indicando encefalopatia generalizada. } \\
\text { Um paciente mostrou atividade sugestiva de estado epiléptico não convulsivo (NCSE). Vinte pacientes foram diagnosticados } \\
\text { com encefalopatia, dois com ANE. Na TC observou-se hipoatenuação difusa, hemorragia parenquimatosa subcortical e } \\
\text { hipodensidades focais em estruturas profundas. Já na RM, envolvimento difuso da substância branca profunda, corpo caloso e } \\
\text { gânglios da base. }\end{array}$ \\
\hline $\begin{array}{l}\text { (Byrnes } \\
\text { 2020) }\end{array}$ & Relato de caso & $\begin{array}{l}\text { Um homem sem-teto de } 36 \text { anos } \\
\text { de idade, saudável, suspeito de } \\
\text { overdose, e confirmado para } \\
\text { COVID-19 }\end{array}$ & $\begin{array}{l}\text { Apresentou fala arrastada e pupilas pontuais. Estava encefalopático com movimentos coreiformes, relacionado à invasão direta } \\
\text { do SNC e a toxicidade autoimune associada a infecção. RM: múltiplas lesões focais na região cortical, subcortical e na } \\
\text { substância periventricular, incluindo o hipocampo, com difusão restrita pontual nos gânglios da base direitos. LCR com } \\
\text { negatividade para vírus ou bactérias e com elevação de leucócitos e dos níveis proteína básica de mielina. Os dados foram } \\
\text { indicativos de ADEM. }\end{array}$ \\
\hline $\begin{array}{l}\text { (Huang } \\
\text { 2020) }\end{array}$ & Relato de caso & $\begin{array}{l}\text { Mulher de } 40 \text { anos com DM2 e } \\
\text { obesidade, internada por } \\
\text { encefalite, confirmada para } \\
\text { COVID-19. }\end{array}$ & $\begin{array}{l}\text { Apresentou quadro de síncope e estado mental alterado. A infecção mostrou-se confinada à região central do SNC sem } \\
\text { envolvimento de outros sistemas. Positividade para SARS-CoV-2 no LCR. Dados consistentes com encefalite viral. }\end{array}$ \\
\hline $\begin{array}{l}\text { (Hepburn et al., } \\
\text { 2020) }\end{array}$ & Relato de caso & $\begin{array}{l}\text { Dois pacientes do sexo } \\
\text { masculino, em idade avançada, } \\
\text { ambos com histórico de doenças } \\
\text { crônicas, confirmados para } \\
\text { COVID-19. }\end{array}$ & $\begin{array}{l}\text { P1: Apresentou dor nos membros, escala do coma de Glasgow de } 14 \text { anos, alterações dos reflexos nos tendões e no nível de } \\
\text { consciência. EEG: três crises focais relacionadas a região centroparietal direita. RM: hiperintensidades crônicas da substância } \\
\text { branca. P2: Apresentou dispneia progressiva e estado mental alterado. EEG: convulsões focais foram capturadas e houve } \\
\text { progressão para status focal epilepticus. TC: hipodensidades na substância branca supratentorial. Desenvolveram dias de } \\
\text { encefalopatia aguda e convulsões encefalográficas. }\end{array}$ \\
\hline $\begin{array}{l}\text { (Kishfy et al., } \\
2020)\end{array}$ & Relato de caso & $\begin{array}{l}\text { Um homem de } 58 \text { anos e uma } \\
\text { mulher de } 67 \text { anos ambos com } \\
\text { histórico médico, confirmados } \\
\text { para COVID-19 }\end{array}$ & $\begin{array}{l}\text { RM e TC revelaram achados compatíveis com o PRES. Em ambos pacientes, observaram-se hiperintensidades envolvendo a } \\
\text { substância branca subcortical nos lobos occiptais e temporais, bem como pontos hemorrágicos. As flutuações da pressão } \\
\text { arterial em ambos também contribuíram para o quadro. }\end{array}$ \\
\hline et al., & Relatos de casos & $\begin{array}{l}6 \text { Pacientes graves com } \\
\text { meningoencefalite autoimune } \\
\text { relacionada ao COVID-19. }\end{array}$ & $\begin{array}{l}\text { Em três pacientes RM mostrou hiperintensidade da substância cortical ou branca, realce de contraste e características } \\
\text { hemorrágicas sulcais, todas consideradas compatíveis com meningoencefalite. LCR negativo para o vírus e com altos níveis de } \\
\text { proteína e albumina sem pleocitose, além de aumento dos marcadores inflamatórios. Dados consistentes de meningoencefalite } \\
\text { autoimune. }\end{array}$ \\
\hline $\begin{array}{l}\text { ( Dixon et al., } \\
\text { 2020) }\end{array}$ & Relato de caso & $\begin{array}{l}\text { Uma mulher de } 59 \text { anos com } \\
\text { antecedentes de anemia aplásica, } \\
\text { dependente de transfusão, } \\
\text { COVID-19 confirmada. }\end{array}$ & $\begin{array}{l}\text { Apresentou convulsões e nível de consciência reduzido. TC: inchaço do tronco cerebral. RM: piora do inchaço com } \\
\text { hemorragia simétrica lesões no tronco encefálico, amígdalas, putamen e núcleos talâmicos. Além disso, houve também } \\
\text { envolvimento da ponte, da medula e de outras regiões em menor grau. Os dados foram consistentes com ANE hemorrágica. }\end{array}$ \\
\hline $\begin{array}{l}\text { (Wong et al., } \\
\text { 2020) }\end{array}$ & Relato de caso & $\begin{array}{l}\text { Homem de } 40 \text { anos, confirmado } \\
\text { para COVID- } 19 .\end{array}$ & $\begin{array}{l}\text { Desenvolveu diplopia, oscilopsia, ataxia nos membros e disfunção aguda do tronco cerebral. O exame clínico revelou fraqueza } \\
\text { facial bilateral leve e movimentos reduzidos da língua. RM do cérebro e da coluna cervical sugeriu uma rombencefalite/mielite } \\
\text { inflamatória. Havia aumento da lesão de sinal no pedúnculo cerebelar inferior direito, envolvendo uma pequena porção da } \\
\text { medula, inchaço no tecido afetado e hemorragia associada. }\end{array}$ \\
\hline
\end{tabular}




\begin{tabular}{|c|c|c|c|}
\hline $\begin{array}{l}\text { (Filatov et al., } \\
\text { 2020) }\end{array}$ & Relato de caso & $\begin{array}{l}\text { Um homem de } 72 \text { anos de idade } \\
\text { com histórico de } \\
\text { hipertensão,hiperlipidemia e } \\
\text { DM2, confirmado com COVID- } \\
19\end{array}$ & $\begin{array}{l}\text { Não respondia a comandos verbais, nem reagia a estímulos dolorosos. RM: pequeno achado incidental de um infarto no córtex } \\
\text { parietal esquerdo. EEG: desaceleração bilateral consistente com encefalopatia e nenhuma atividade epileptiforme evidenciada. } \\
\text { LCR sem evidências de infecção ativa. Dados consistentes com encefalopatia. }\end{array}$ \\
\hline $\begin{array}{l}\text { (Bernard-valnet } \\
\text { et al., 2020) }\end{array}$ & Relato de caso & $\begin{array}{l}\text { Duas pacientes com mais de } 60 \\
\text { anos, infectadas com SARS- } \\
\text { CoV-2 }\end{array}$ & $\begin{array}{l}\text { P1: Apresentou desorientação, forte déficit de atenção, sintomas psicóticos agudos e uma tonic clonic seizure. EEG revelou } \\
\text { non-convulsive, focal status epilepticus. Sem evidência de infecção no LCR. P2: apresentou fortes dores de cabeça e } \\
\text { desorientação. LCR com negatividade para patógeno viral/bacteriano e com pleocitose linfocítica. Apesar RM cerebral normal } \\
\text { em ambos, a punção lombar foi indicativa de meningoencefalite viral. }\end{array}$ \\
\hline $\begin{array}{l}\text { (Franceschi et al., } \\
\text { 2020) }\end{array}$ & Relato de caso & $\begin{array}{l}\text { Dois pacientes confirmados com } \\
\text { COVID-19. Homem de } 48 \text { anos, } \\
\text { obeso, sem histórico médico. } \\
\text { Mulher de } 67 \text { anos e múltiplas } \\
\text { comorbidades. }\end{array}$ & $\begin{array}{l}\text { Ambos apresentaram estado mental alterado. Foram encontrados edemas principalmente nas regiões parieto-occipitais na TC e } \\
\text { RM dos dois casos. Enquanto no SWI foram observadas hemorragias em diferentes áreas. Os dados foram compatíveis com } \\
\text { PRES hemorrágico. }\end{array}$ \\
\hline $\begin{array}{l}\text { (Karimi et al., } \\
\text { 2020) }\end{array}$ & Relato de caso & $\begin{array}{l}\text { Mulher de } 30 \text { anos, } \\
\text { histórico sem } \\
\text { confirmação de COVIDico, } 19 \text {. }\end{array}$ & $\begin{array}{l}\text { Desenvolveu convulsão tônico-clônica generalizada. No exame neurológico, apresentou-se sonolenta com desorientação ao } \\
\text { longo do tempo. Após internação, teve várias crises convulsivas. RM do cérebro normal e LCR sem evidência de infecção. Os } \\
\text { dados são compatíveis com encefalite viral. }\end{array}$ \\
\hline $\begin{array}{l}\text { (Sohal \& } \\
\text { Mansur, 2020) }\end{array}$ & Relato de caso & $\begin{array}{l}\text { Homem de } 72 \text { anos com história } \\
\text { de hipertensão, doença arterial } \\
\text { coronariana, DM2, doença renal, } \\
\text { em hemodiálise, confirmado } \\
\text { para COVID-19. }\end{array}$ & $\begin{array}{l}\text { Apresentou estado mental alterado, com múltiplos episódios de convulsão com movimentos tônicos-clônicos das extremidades } \\
\text { superior e inferior. EEG de } 24 \text { horas mostrou six left temporal seizures e ondas agudas temporais esquerdas que eram } \\
\text { epileptogênicas. Posteriormente, foram observados movimentos tônicos de suas extremidades superiores } 2-3 \text { vezes ao dia. Os } \\
\text { achados foram consistentes com encefalite. }\end{array}$ \\
\hline $\begin{array}{l}\text { (Radmanesh et } \\
\text { al., 2020) }\end{array}$ & $\begin{array}{l}\text { Estudo } \\
\text { restrospectivo } \\
\text { entre } 5 \text { e } 25 \text { de } \\
\text { abril de } 2020\end{array}$ & $\begin{array}{l}11 \text { pacientes gravemente } \\
\text { enfermos com COVID-19 e } \\
\text { estado mental persistentemente } \\
\text { deprimido. }\end{array}$ & $\begin{array}{l}\text { Os } 11 \text { pacientes foram submetidos a RM, dos quais havia } 4 \text { pacientes com apenas leucoencefalopatia difusa, } 1 \text { com apenas } \\
\text { micro-hemorragias pontuais e } 6 \text { com uma combinação de ambos. Pacientes com leucoencefalopatia apresentaram } \\
\text { hiperintensidades em T2 simétricas e confluentes, com difusão restrita leve envolvendo substância branca profunda e } \\
\text { subcortical bilateral; micro-hemorragias pontuais com envolvimento predominante da substância branca justacortical e calosa } \\
\text { foram encontradas em } 7 \text { pacientes. }\end{array}$ \\
\hline $\begin{array}{l}\text { (Parsons et al., } \\
\text { 2020) }\end{array}$ & Relato de caso & $\begin{array}{l}\text { Paciente do sexo feminino com } \\
51 \text { anos de idade, confirmada } \\
\text { para COVID-19. }\end{array}$ & $\begin{array}{l}\text { Paciente apresentou coma, resposta oculocefálica unilateral comprometida e hemiparesia esquerda. RM demonstrou lesões } \\
\text { hiperintensas dispersas na imagem FLAIR na substância branca hemisférica profunda e justacortical. LCR demonstrou red } \\
\text { blood cells com xantocromia, leve aumento de proteína e quatro bandas oligoclonais, presentes também no soro. RM, LCR e } \\
\text { curso clínico foram consistentes com ADEM. }\end{array}$ \\
\hline (Efe et al., 2020) & Relato de caso & $\begin{array}{l}\text { Paciente do sexo feminino, } 35 \\
\text { anos de idade, internada com dor } \\
\text { de cabeça, náusea, tontura e } \\
\text { convulsões refratárias a } \\
\text { medicamentos, confirmada para } \\
\text { COVID-19 no pós-operatório. }\end{array}$ & $\begin{array}{l}\text { A RM mostrou lesão hiperintensa no lobo temporal esquerdo em T2 FLAIR. Espectroscopia por RM demonstrou um pico } \\
\text { elevado de colina, juntamente com uma diminuição do pico de N-acetilaspartato, sugestivo de glioma de alto grau, porém o } \\
\text { exame histopatológico revelou encefalite, com infiltração linfocítica concêntrica nos espaços perivasculares, causando dano } \\
\text { neuronal e alterações hipóxicas difusas no parênquima cerebral circundante. }\end{array}$ \\
\hline $\begin{array}{l}\text { (Al-Olama et al., } \\
\text { 2020) }\end{array}$ & Relato de caso & $\begin{array}{l}\text { Paciente do sexo masculino, } 36 \\
\text { anos de idade, internado em } \\
\text { caráter de emergência. Positivo } \\
\text { para SARS-CoV-2 no LCR. }\end{array}$ & $\begin{array}{l}\text { Apresentação clínica neurológica incluía sonolência e confusão leve. Na TC foram observados pontos de hemorragia e } \\
\text { hematomas cercados de edemas. Houve deteção aumentada de bilateral supratentorial leptomeningeal, o que apoiou ainda } \\
\text { mais o diagnóstico de meningoencefalite relacionada à COVID-19. Foi encontrado RNA de SARS-CoV-2 apenas no LCR. }\end{array}$ \\
\hline $\begin{array}{l}\text { (Hayashi et al., } \\
\text { 2020) }\end{array}$ & Relato de caso & $\begin{array}{l}\text { Paciente do sexo masculino, } 75 \\
\text { anos de idade com histórico de } \\
\text { doença de Alzheimer leve, } \\
\text { confirmado para COVID-19. }\end{array}$ & $\begin{array}{l}\text { O exame neurológico revelou consciência alerta e movimento normal dos olhos, mas o teste dedo-no-nariz mostrou dismetria } \\
\text { bilateral acentuada. Observou-se marcha atáxica leve. A RM revelou hiperintensidade anormal no esplênio do corpo caloso em } \\
\text { difusão, suspeita de encefalite clinicamente leve/encefalopatia com lesão esplênica reversível - mild encephalitis reversible. } \\
\text { Apresentou perturbação transitória da consciência leve. }\end{array}$ \\
\hline
\end{tabular}




\begin{tabular}{|c|c|c|c|}
\hline $\begin{array}{l}\text { (Moriguchi et al., } \\
\text { 2020) }\end{array}$ & elato de caso & $\begin{array}{l}\text { Paciente do sexo masculino, } 24 \\
\text { anos de idade, com convulsão } \\
\text { acompanhada de inconsciência. } \\
\text { Positivo para SARS-CoV-2 no } \\
\text { LCR. }\end{array}$ & $\begin{array}{l}\text { O paciente apresentou convulsões generalizadas transitórias e rigidez no pescoço. RM mostrou hiperintensidade ao longo da } \\
\text { parede do ventrículo lateral direito. Imagens de FLAIR mostraram alterações de sinal hiperintensas no lobo temporal mesial } \\
\text { direito e hipocampo, com ligeira atrofia hipocampal sugerindo a possibilidade de meningite/encefalite por SARS-CoV-2. O } \\
\text { RNA específico de SARS-CoV-2 foi detectado apenas no LCR. }\end{array}$ \\
\hline $\begin{array}{l}\text { (Zayet et al., } \\
2020)\end{array}$ & Relato de caso & $\begin{array}{l}\text { Dois pacientes, ambos do sexo } \\
\text { masculino, um com } 68 \text { anos e o } \\
\text { outro com } 39 \text { anos, ambos } \\
\text { confirmados para COVID-19. }\end{array}$ & $\begin{array}{l}\text { P1: apresentou resposta verbal confusa e escore de coma, a partir do escore de Glasgow, de 14/15. P2: apresentou disartria, } \\
\text { desatenção, sonolência progressiva e diminuição da consciência. O exame neurológico mostrou uma pontuação na escala de } \\
\text { coma de Glasgow de } 12 / 15 \text { com afasia não fluente. Ambas RM estavam normais e LCR negativo para SARS-CoV-2. Dados } \\
\text { compatíveis com encefalopatia. }\end{array}$ \\
\hline $\begin{array}{l}\text { (Pilotto } \\
\text { 2020) }\end{array}$ & & $\begin{array}{l}\text { Paciente do sexo masculino, } 60 \\
\text { anos de idade, com alteração } \\
\text { grave de consciência, } \\
\text { confirmado para COVID-19 }\end{array}$ & $\begin{array}{l}\text { Foram detectados reflexos palmomentais e glabella positivos com rigidez nucal moderada, sem sinais focais no exame } \\
\text { neurológico. Análise do LCR apresentou achados inflamatórios, com leve pleocitose linfocítica e aumento moderado de } \\
\text { proteína. O RT-PCR no LCR para SARS-CoV-2 também foi negativo. EEG exibiu lentidão generalizada, com menor } \\
\text { reatividade aos estímulos acústicos. Com o curso da infecção, a análise do LCR revelou concentrações aumentadas de } \\
\text { interleucina (IL) } 6 \text {, IL-8, fator de necrose tumoral alfa (TNF- } \alpha \text { ) e } \beta 2 \text {-microglobulina. Dados compatíveis com encefalite. }\end{array}$ \\
\hline $\begin{array}{l}\text { (Deliwala et al., } \\
\text { 2020) }\end{array}$ & Relato de caso & $\begin{array}{l}\text { Mulher de } 31 \text { anos, previamente } \\
\text { saudável, confirmada para } \\
\text { COVID-19. }\end{array}$ & $\begin{array}{l}\text { Paciente exibia características de confusão e encefalopatia. Os dímeros em série aumentaram nos dias que antecederam o } \\
\text { acidente vascular cerebral, indicando estado de hipercoagulabilidade. TC revelou alterações isquêmicas na região suprida pela } \\
\text { artéria cerebral média direita consistente com um acidente vascular cerebral cortical. }\end{array}$ \\
\hline $\begin{array}{l}\text { (Benameur et al., } \\
\text { 2020) }\end{array}$ & & $\begin{array}{l}\text { Três pacientes: } 1 \text { (uma) do sexo } \\
\text { feminino, de } 31 \text { anos de idade e } \\
2 \text { (dois) do sexo masculino de } 34 \\
\text { e } 64 \text { anos de idade. Todos } \\
\text { confirmados para COVID-19. }\end{array}$ & $\begin{array}{l}\text { A neuroimagem mostrou alterações unilaterais, bilaterais e da linha média sem melhora, que não são prontamente atribuíveis a } \\
\text { causas vasculares. A análise do LCR nos três pacientes apresentou níveis aumentados de IgM anti-S1 e de IL-6, IL-8 e IL-10. } \\
\text { SARS-CoV-2 não foi identificado em nenhuma amostra do LCR. Achados consistentes com encefalite e encefalopatia }\end{array}$ \\
\hline $\begin{array}{l}\text { (Poyiadji et al., } \\
\text { 2020) }\end{array}$ & Relato de caso & $\begin{array}{l}\text { Paciente do sexo feminino, } 49 \\
\text { anos de idade, com confirmação } \\
\text { para COVID- } 19 .\end{array}$ & $\begin{array}{l}\text { A TC sem contraste demonstrou hipoatenuação simétrica dentro do thalami medial bilateral com angiograma de TC normal e } \\
\text { venograma de TC. A RM apresentava lesões que aumentam a borda hemorrágica dentro do tálamo bilateral, lobos temporais } \\
\text { mediais e regiões subinsulares. As lesões aparecem hipoatenuantes nas imagens de TC e a RM demonstra sinal hiperintenso T2 } \\
\text { FLAIR com hemorragia interna. Os dados foram consistentes com ANE. }\end{array}$ \\
\hline et al., & $\begin{array}{l}\text { Estudo de série de } \\
\text { casos }\end{array}$ & $\begin{array}{l}\text { Quatro pacientes idosos com } \\
\text { delirium atendidos em hospital } \\
\text { geral nos Estados Unidos com } \\
\text { históricos de distúrbios } \\
\text { neurocognitivos } \\
\text { comportamentais. } \\
\text { confirmados para COVID-19 }\end{array}$ & $\begin{array}{l}\text { P1: TC não apresentou resultados significativos. P2: EEG mostrou desaceleração teta difusa, descargas generalizadas isoladas } \\
\text { de baixa e frequente amplitude e breves períodos frequentes de atividade delta rítmica generalizada de } 1 \mathrm{~Hz} \text { com contorno } \\
\text { acentuado e predominância bifrontal. } \\
\text { P3: TC demonstrou leve perda de volume, alterações isquêmicas leves de pequenos vasos e um hematoma subdural agudo } \\
\text { sobre crônico de } 7 \text { milímetros }(\mathrm{mm}) \text { do lado esquerdo sobre os lobos frontal, parietal e temporal. EEG mostrou desaceleração } \\
\text { difusa do delta irregular e corridas frequentes de descargas epileptiformes periódicas generalizadas. A mioclonia multifocal foi } \\
\text { observada em todos, exceto um paciente. Dados consistentes como encefalopatia. }\end{array}$ \\
\hline
\end{tabular}

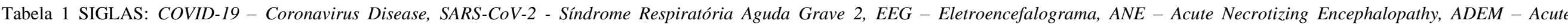

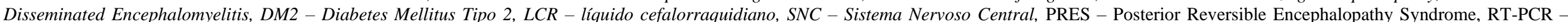

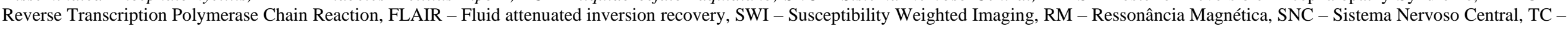
Tomografia Computadorizada.

A maior parte dos artigos realizou hemoculturas ou painel viral/bacteriano na busca por outros patógenos.

Fonte: Autores. 


\section{Discussão}

Todos os pacientes foram testados como positivos para SARS-CoV-2 pela RT-PCR com a coleta de material por $s w a b$ das vias respiratórias ou a partir do isolamento do RNA viral no LCR. A maior parte dos relatados apresentavam apenas sintomas comuns da COVID-19 de forma leve (febre, tosse seca, fraqueza) e com opacidades na tomografia computadorizada (TC) do tórax. Outros, porém, evoluíam para quadros mais graves com insuficiência respiratória (Huang et al., 2020; Kishfy et al., 2020; Sohal \& Mansur, 2020; Hayashi et al., 2020) e até mesmo acometimento de outros sistemas (Kishfy et al., 2020; Wong et al., 2020).

As encefalites/encefalopatias associadas a COVID-19, assim como em outras encefalopatias, apresentam-se clinicamente com confusão, agitação, delírio e coma (Garg et al., 2020). Existem casos em que as manifestações neurológicas são precedidas por dias de sintomas respiratórios (Pilotto et al., 2020; Beach et al., 2020) e em outros, apresentam-se como os únicos sintomas nos pacientes (Huang et al., 2020; Hayashi et al., 2020), como já relatado em estudo anterior (Ahmad \& Rathore, 2020).

Os exames neurológicos realizados que mais contribuíram para definir o diagnóstico nos casos relatados incluem a TC e a ressonância magnética (RM). Foram comuns os achados de hiperintensidades da substância branca, cortical ou subcortical, e hipodensidades e hipoatenuação de estruturas profundas da substância branca. Também puderam ser observadas múltiplas lesões envolvendo hipocampo, núcleos de base, medula, tronco encefálico, tálamo, dentre outras regiões, tendo pontos hemorrágicos muitas vezes associados.

O envolvimento do SNC no contexto da COVID-19 pode ser verdadeiramente neurológico, uma consequência de lesão sistêmica ou uma combinação de ambos. A atividade neurotrópica do SARS-CoV-2 é apoiada pela observação da disseminação viral no LCR (Munhoz et al., 2020). Vários estudos realizaram sua avaliação em busca da presença do vírus ou de alterações na amostra que fossem compatíveis com infecção. O RNA viral pôde ser detectado na amostra de LCR apenas em alguns estudos (Moriguchi et al., 2020; Huang et al., 2020; Poyiadji et al., 2020), e apenas no primeiro houve positividade também no swab das vias respiratórias. A maioria das buscas realizadas não identificaram o vírus no LCR, o que não exclui a possibilidade de acometimento do SNC. Isso porque a carga viral pode ser insuficiente para detecção, uma vez que a disseminação do vírus tem caráter transitório e sua quantidade no LCR pode ser extremamente baixa (Ye et al., 2020).

Além da presença do vírus em si, outros achados no LCR contribuíram com a suspeita de infecção viral ativa. Podem ser observados marcadores inflamatórios, níveis aumentados de proteínas ou células de defesa (Garg et al., 2020). Níveis aumentados de interleucinas (IL) 6, IL-8 e IL-10, tumor necrosis factor alpha (TNF- $\alpha$ ) e de IgM anti-S1 e IgM anti-E em alguns pacientes com complicações neurológicas foram identificadas no LCR (Pilotto et al., 2020; Benameur et al., 2020), os quais são consistentes com o envolvimento direto no SNC, pois fornecem indícios de alterações inflamatórias peri-infecciosas e pós-infeciosas do LCR durante o curso da doença (Benameur et al., 2020).

A ocorrência de meningoencefalite também deve ser lembrada como um possível dano cerebral resultante da COVID19. Um dos estudos de meningoencefalite autoimune com seis pacientes graves identificou um coeficiente de albumina elevado no LCR da maior parte deles e altos níveis de proteína em todos eles, bem como o aumento de marcadores inflamatórios (ferritina) e inflamação cerebral bilateral mostrada na RM compatível com a doença (Dogan et al., 2020). Em outro estudo, exames de imagem revelaram achados consistentes com meningoencefalite complicada por hematoma intracerebral e hematoma subdural (Al-olama et al., 2020). Um tipo específico de encefalite foi associado à COVID-19, após Hayashi e colaboradores (Hayashi et al., 2020) descreverem o primeiro caso presuntivo de mild encephalitis/encephalopathy with a reversible splenial lesion em um paciente sem sintomas respiratórios, porém com diagnóstico de ataxia cerebelar e lesão 
esplênica do corpo caloso. Além disso, o primeiro caso de encefalite mimicking um tumor glial foi relatado recentemente, a partir da confirmação histopatológica em um paciente infectado (Efe et al., 2020).

Alguns relatos também documentaram uma possível associação da COVID-19 com o desenvolvimento da Acute Disseminated Encephalomyelitis (ADEM) (Byrnes et al., 2020; Parsons et al., 2020). Trata-se de uma doença autoimune e desmielinizante rara, geralmente pós-infecciosa, que causa encefalopatia e déficits multifocais neurológicos. O tronco cerebral cortical da substância branca, os nervos ópticos e a medula espinhal sofrem alterações variáveis e na RM geralmente apresentam-se lesões multifocais generalizadas e difusas da substância branca T2 / FLAIR (Garg et al., 2020). Apesar de existirem outras causas relacionadas a esses achados como leucoencefalopatia tóxica, metabólicos e trauma, elas não eram compatíveis, visto que os exames clínicos e neurológicos foram consistentes com um evento desmielinizante agudo (Byrnes et al., 2020). Além disso, resultados a partir da análise LCR também corroboram com o surgimento da ADEM diante da observação da proteína básica da mielina (Byrnes et al., 2020). O vírus reage de maneira cruzada com essa proteína sugerindo mimetismo molecular e um potencial patogênese para lesão do SNC (Scullen et al., 2020). Sua presença é um sinal da quebra ativa da mielina, aparecendo comumente elevada na ADEM (Byrnes et al., 2020).

Nos estudos selecionados, vários pacientes tiveram episódios de convulsões e resultados de eletroencefalograma (EEG) compatíveis com convulsões focais e status epilepticus convulsivo e não convulsivo (Moriguchi et al., 2020; Scullen et al., 2020; Hepburn et al., 2020; Dixon et al., 2020; Bernard-Valnet et al., 2020; Karimi et al., 2020; Sohal \& Mansur, et al., 2020). Já existe na literatura uma associação entre episódios convulsivos e o envolvimento do SNC na COVID-19 (Durjoy \& Alfredo, 2020). Demonstrou-se anteriormente para outros a proliferação de outros CoVs promovem uma tempestade de citocinas pró-inflamatórias que leva a quebra da barreira hematoencefálica, especialmente IL-8 e proteína quimioatraente de monócitos-1 (MCP-1) (Li et al., 2017; Deshmane et al., 2009). É possível então que após a penetração da barreira hematoencefálica, SARS-CoV-2 retarde a microcirculação cerebral, por meio da criação de um estado hipercoagulável, permitindo o aumento da interação do vírus com os receptores endoteliais e receptores do tecido glial, podendo predispor os pacientes a convulsões (Hepburn et al., 2020).

Essa ligação do vírus à ECA-2 nos vasos sanguíneos cerebrais pode aumentar a pressão luminal neles, e, juntamente com o enfraquecimento da camada endotelial e da barreira hematoencefálica, resultam em disfunção da autorregulação da circulação cerebral (Kishfy et al., 2020) e provocam hemorragias intracerebrais (Durjoy \& Alfredo, 2020). A disfunção endotelial parece ter contribuído para os casos relatados de Posterior reversible encephalophaty syndrome (PRES), visto que pacientes ficaram intubados por períodos prolongados com instabilidades nas pressões sanguíneas (Kishfy et al., 2020). Comumente a neuroimagem da PRES demonstra edema vasogênico, sobretudo, nos lobos parieto-occipitais, podendo também estar presente em "watershed distributions", lobos frontais, lobos temporais inferiores, gânglios basais, no tronco cerebral e cerebelo. Além disso, podem incluir hemorragias petéquias e hematomas intraparenquimatosos (Franceschi et al., 2020).

Outro fator que pode estar relacionado ao desenvolvimento das lesões cerebrais e convulsões observadas relaciona-se com a hipoxemia grave decorrente da infecção (Durjoy \& Alfredo, 2020). A hipóxia no cérebro causa leva à vasodilatação cerebral, inchaço dos neurônios, edema intersticial e obstrução do fluxo sanguíneo cerebral (Kaya et al., 2020).

Foram também verificados casos de acute necrotizing encephalopathy (ANE) (Scullen et al., 2020; Dixon et al., 2020; Poyiadji et al., 2020) Essa condição já foi associada anteriormente a outros vírus (Mizuguchi et al., 2020) e consiste em um distúrbio raro associado à disfunção cerebral que pode levar a convulsões, problemas hepáticos e desorientação mental após infecção principalmente viral (Das et al., 2020). A neuroinflamação resulta de uma tempestade de citocinas caracterizada principalmente pela produção de IL-6 (Bridwell; Das et., 2020) e danos à barreira hematoencefálica (Rossi, 2008). Além de causar encefalopatia grave no paciente, pode também resultar em derrame (Das et al., 2020) e se apresentar como um sinal 
sentinela para desenvolvimento de acidente vascular cerebral (AVC) em pacientes com COVID-19 (Deliwala et al., 2020) Um caso de ANE hemorrágica também foi relacionado à infecção, sendo comumente caracterizada por múltiplas lesões simétricas no tálamo, estriado, substância branca cerebral, tronco cerebral e aumento de proteína no LCR e frequentemente de evolução rápida com convulsões, consciência reduzida e vômitos (Dixon et al., 2020).

Alguns pacientes também desenvolveram delirium com uma mudança de estado mental como sintoma de apresentação (Beach et al., 2020). Os sintomas, em alguns casos, evoluíram para alogia progressiva inicialmente com falta de fala e progressão para mutismo. O delirium é um distúrbio de curso flutuante e início agudo que afeta a atenção e a consciência, cuja manifestação é comum em encefalopatias leve a moderada (Garg et al., 2020). Alterações motoras como mioclonia multifocal nas extremidades inferiores e superiores bilaterais se apresentaram em alguns dos pacientes descritos, além do aumento do tônus muscular e rigidez, o qual pode estar relacionado a uma resposta inflamatória autoimune induzida por vírus neurotrópicos a antígenos dos gânglios de base (Beach et al., 2020).

Apesar de ser uma doença muito nova, cujos conhecimentos sobre sua patogênese ainda não estão totalmente elucidados, cada vez mais as alterações neurológicas desenvolvidas em pacientes com a COVID-19 estão sendo associadas à atuação do vírus no organismo humano. Em um estudo pós-morte já foi revelada a presença de partículas virais de SARSCoV-2 no endotélio cerebral e capilar, evidência de que o vírus pode se propagar diretamente para o tecido cerebral (PanizMondolfi et al., 2020). Além disso, observou-se edema cerebral em necropsias de pacientes acometidos por COVID-19 (Xu et al., 2020). Esse envolvimento do SNC associado à COVID-19 é considerado indicador de prognóstico ruim (Li et al., 2020; Mao et al., 2020), podendo levar a rápida deterioração clínica dos pacientes (Byrnes et al., 2020).

\section{Conclusão}

A presente revisão visa contribuir para o conhecimento do impacto neurológico dos coronavírus, que pode promover encefalites e encefalopatias em pacientes com a COVID-19. Assim, é importante que os profissionais de saúde estejam preparados para o acompanhamento dos pacientes com complicações neurológicas, visto a gravidade e heterogeneidade das características clínicas e laboratoriais. Ainda não está elucidado qual a incidência real de complicações neurológicas, suas gravidades.

Há, portanto, a necessidade de mais estudos epidemiológicos e pesquisas futuras, para esclarecer essas lacunas em nosso conhecimento atual, bem como estudos de investigação dos mecanismos e consequências emergentes do envolvimento neurológico do SARS-CoV-2 e trabalhos que tragam uma perspectiva sobre os efeitos neuroanatômicos e cognitivos associados às complicações da COVID-19 no encéfalo, tendo em vista a natureza nova da doença e seus impactos sociais a longo prazo.

\section{Referências}

Ahmad, I., \& Rathore, F. A. (2020). Neurological manifestations and complications of COVID-19: a literature review. Journal of Clinical Neuroscience, 77, 8-12.

Al-Olama, M., Rashid, A., \& Garozzo, D. (2020). COVID-19-associated meningoencephalitis complicated with intracranial hemorrhage: a case report. Acta neurochirurgica, 162(7), 1495-1499.

Beach, S. R., Praschan, N. C., Hogan, C., Dotson, S., Merideth, F., Kontos, N., \& Smith, F. A. (2020). Delirium in COVID-19: a case series and exploration of potential mechanisms for central nervous system involvement. General Hospital Psychiatry, 65, 47-53.

Benameur, K., Agarwal, A., Auld, S. C., Butters, M. P., Webster, A. S., Ozturk, T., \& Hu, W. T. (2020). Encephalopathy and encephalitis associated with cerebrospinal fluid cytokine alterations and coronavirus disease, Atlanta, Georgia, USA, 2020. Emerging infectious diseases, 26(9), 2016. 
Research, Society and Development, v. 10, n. 12, e464101220764, 2021 (CC BY 4.0) | ISSN 2525-3409 | DOI: http://dx.doi.org/10.33448/rsd-v10i12.20764

Bernard-Valnet, R., Pizzarotti, B., Anichini, A., Demars, Y., Russo, E., Schmidhauser, M., \& Du Pasquier, R. (2020). Two patients with acute meningoencephalitis concomitant with SARS-CoV-2 infection. European journal of neurology., 0-1.

Bonzano, C., Borroni, D., Lancia, A., \& Bonzano, E. (2020). Doxycycline: from ocular rosacea to COVID-19 anosmia. New insight into the coronavirus outbreak. Frontiers in medicine, 7, 200.

Bridwell, R., Long, B., \& Gottlieb, M. (2020). Neurologic complications of COVID-19. The American journal of emergency medicine, 38(7), 1549-e3.

Byrnes, S., Bisen, M., Syed, B., Huda, S., Siddique, Z., Sampat, P., \& Dargon, I. (2020). COVID-19 encephalopathy masquerading as substance withdrawal. Journal of medical virology, 6-8.

Carod-Artal, F. J. (2020). Complicaciones neurológicas por coronavirus y COVID-19. Rev Neurol, 70(9), $311-322$.

Condie, L. O. (2020). Neurotropic mechanisms in COVID-19 and their potential influence on neuropsychological outcomes in children. Child Neuropsychology, 26(5), 577-596.

Danser, A. J., Epstein, M., \& Batlle, D. (2020). Renin-angiotensin system blockers and the COVID-19 pandemic: at present there is no evidence to abandon renin-angiotensin system blockers. Hypertension, 75(6), 1382-1385.

Das, G., Mukherjee, N., \& Ghosh, S. (2020). Neurological insights of COVID-19 pandemic. ACS chemical neuroscience, 11(9), 1206-1209.

Deliwala, S., Abdulhamid, S., Abusalih, M. F., Al-Qasmi, M. M., \& Bachuwa, G. (2020). Encephalopathy as the sentinel sign of a cortical stroke in a patient infected with coronavirus disease-19 (COVID-19). Cureus, 12(5).

Deshmane, S. L., Kremlev, S., Amini, S., \& Sawaya, B. E. (2009). Monocyte chemoattractant protein-1 (MCP-1): an overview. Journal of interferon \& cytokine research, 29(6), 313-326.

Dixon, L., Varley, J., Gontsarova, A., Mallon, D., Tona, F., Muir, D., \& Everitt, A. (2020). COVID-19-related acute necrotizing encephalopathy with brain stem involvement in a patient with aplastic anemia. Neurology-Neuroimmunology Neuroinflammation, 7(5), 1-8.

Dogan, L., Kaya, D., Sarikaya, T., Zengin, R., Dincer, A., Akinci, I. O., \& Afsar, N. (2020). Plasmapheresis treatment in COVID-19-related autoimmune meningoencephalitis: case series. Brain, behavior, and immunity, 87, 155-158.

Durjoy, L., \& Alfredo, A. (2020). COVID-19 Pandemic: A Neurological Perspective. Cureus, 12(4).

Efe, I. E., Aydin, O. U., Alabulut, A., Celik, O., \& Aydin, K. (2020). COVID-19- associated encephalitis mimicking glial tumor. World neurosurgery, 140, 46-48.

Filatov, A., Sharma, P., Hindi, F., \& Espinosa, P. S. (2020). Neurological complications of coronavirus disease (COVID-19): encephalopathy. Cureus, 12(3).

Franceschi, A. M., Ahmed, O., Giliberto, L., \& Castillo, M. (2020). Hemorrhagic posterior reversible encephalopathy syndrome as a manifestation of COVID19 infection. American Journal of Neuroradiology, 41(7), 1173-1176.

Garg, R. K., Paliwal, V. K., \& Gupta, A. (2021). Encephalopathy in patients with COVID-19: a review. Journal of Medical Virology, 93(1), $206-222$.

Hayashi, M., Sahashi, Y., Baba, Y., Okura, H., \& Shimohata, T. (2020). COVID-19-associated mild encephalitis/encephalopathy with a reversible splenial lesion. Journal of the neurological sciences, 415, 116941.

Hepburn, M., Mullaguri, N., George, P., Hantus, S., Punia, V., Bhimraj, A., \& Newey, C. R. (2021). Acute symptomatic seizures in critically ill patients with COVID-19: is there an association? Neurocritical care, 34, 139-143.

Huang, Y. H., Jiang, D., \& Huang, J. T. (2020). SARS-CoV-2 detected in cerebrospinal fluid by PCR in a case of COVID-19 encephalitis. Brain, behavior, and immunity, 87, 149.

Hung, E. C., Chim, S. S., Chan, P. K., Tong, Y. K., Ng, E. K., Chiu, R. W., \& Lo, Y. D. (2003). Detection of SARS coronavirus RNA in the cerebrospinal fluid of a patient with severe acute respiratory syndrome. Clinical Chemistry, 49(12), 2108.

Karimi, N., Razavi, A. S., \& Rouhani, N. (2020). Frequent convulsive seizures in an adult patient with COVID-19: a case report. Iranian Red Crescent Medical Journal, 22(3).

Kaya, Y., Kara, S., Akinci, C., \& Kocaman, A. S. (2020). Transient cortical blindness in COVID-19 pneumonia; a PRES-like syndrome: case report. Journal of the neurological sciences, 413, 116858 .

Kishfy, L., Casasola, M., Banankhah, P., Parvez, A., Jan, Y. J., Shenoy, A. M., \& AbdelRazek, M. A. (2020). Posterior reversible encephalopathy syndrome (PRES) as a neurological association in severe Covid-19. Journal of the neurological sciences, 414, 116943.

Klok, F. A., Kruip, M. J. H. A., Van der Meer, N. J. M., Arbous, M. S., Gommers, D. A. M. P. J., Kant, K. M., \& Endeman, H. (2020). Incidence of thrombotic complications in critically ill ICU patients with COVID-19. Thrombosis research, 191, 145-147.

Kotfis, K., Roberson, S. W., Wilson, J. E., Dabrowski, W., Pun, B. T., \& Ely, E. W. (2020). COVID-19: ICU delirium management during SARS-CoV-2 pandemic. Critical care, 24(1), 1-9.

Li, H., Xue, Q., \& Xu, X. (2020). Involvement of the nervous system in SARS-CoV-2 infection. Neurotoxicity research, 1. 
Li, Y. C., Bai, W. Z., \& Hashikawa, T. (2020). The neuroinvasive potential of SARS-CoV2 may play a role in the respiratory failure of COVID-19 patients. Journal of medical virology, 92(6), 552-555.

Li, Y., Li, H., Fan, R., Wen, B., Zhang, J., Cao, X., \& Liu, W. (2017). Coronavirus infections in the central nervous system and respiratory tract show distinct features in hospitalized children. Intervirology, 59(3), 163-169.

Linares-Espinós, E., Hernández, V., Domínguez-Escrig, J. L., Fernández-Pello, S., Hevia, V., Mayor, J., \& Ribal, M. J. (2018). Methodology of a systematic review. Metodología de una revisión sistemática. Actas urologicas espanolas, 42(8), 499-506.

Lu, R., Zhao, X., Li, J., Niu, P., Yang, B., Wu, H., \& Tan, W. (2020). Genomic characterisation and epidemiology of 2019 novel coronavirus: implications for virus origins and receptor binding. The lancet, 395(10224), 565-574.

Mao, L., Wang, M., Chen, S., He, Q., Chang, J., Hong, C., \& Hu, B. (2020). Neurological manifestations of hospitalized patients with COVID-19 in Wuhan, China: a retrospective case series study. MedRxiv.

Mehta, P., McAuley, D. F., Brown, M., Sanchez, E., Tattersall, R. S., \& Manson, J. J. (2020). COVID-19: consider cytokine storm syndromes and immunosuppression. The lancet, 395(10229), 1033-1034.

Mizuguchi, M., Yamanouchi, H., Ichiyama, T., \& Shiomi, M. (2007). Acute encephalopathy associated with influenza and other viral infections. Acta Neurologica Scandinavica, 115, 45-56.

Moriguchi, T., Harii, N., Goto, J., Harada, D., Sugawara, H., Takamino, J., \& Shimada, S. (2020). A first case of meningitis/encephalitis associated with SARS-Coronavirus-2. International journal of infectious diseases, $94,55-58$.

Munhoz, R. P., Pedroso, J. L., Nascimento, F. A., Almeida, S. M. D., Barsottini, O. G. P., Cardoso, F. E. C., \& Teive, H. A. G. (2020). Neurological complications in patients with SARS-CoV-2 infection: a systematic review. Arquivos de Neuro-Psiquiatria, 78, 290-300.

Netland, J., Meyerholz, D. K., Moore, S., Cassell, M., \& Perlman, S. (2008). Severe acute respiratory syndrome coronavirus infection causes neuronal death in the absence of encephalitis in mice transgenic for human ACE2. Journal of virology, 82(15), 7264-7275.

Paniz-Mondolfi, A., Bryce, C., Grimes, Z., Gordon, R. E., Reidy, J., Lednicky, J., \& Fowkes, M. (2020). Central nervous system involvement by severe acute respiratory syndrome coronavirus-2 (SARS-CoV-2). Journal of medical virology, 92(7), 699-702.

Parsons, T., Banks, S., Bae, C., Gelber, J., Alahmadi, H., \& Tichauer, M. (2020). COVID-19-associated acute disseminated encephalomyelitis (ADEM). Journal of neurology, 267, 2799-2802.

Pilotto, A., Odolini, S., Masciocchi, S., Comelli, A., Volonghi, I., Gazzina, S., \& Padovani, A. (2020). Steroid-responsive encephalitis in coronavirus disease 2019. Annals of neurology, 88(2), 423-427.

Poyiadji, N., Shahin, G., Noujaim, D., Stone, M., Patel, S., \& Griffith, B. (2020). COVID-19-associated acute hemorrhagic necrotizing encephalopathy: imaging features. Radiology, 296(2), E119-E120.

Radmanesh, A., Derman, A., Lui, Y. W., Raz, E., Loh, J. P., Hagiwara, M., \& Fatterpekar, G. M. (2020). COVID-19-associated diffuse leukoencephalopathy and microhemorrhages. Radiology, 297(1), E223-E227.

Romero-Sánchez, C. M., Díaz-Maroto, I., Fernández-Díaz, E., Sánchez-Larsen, Á., Layos-Romero, A., García-García, J., \& Segura, T. (2020). Neurologic manifestations in hospitalized patients with COVID-19: the ALBACOVID registry. Neurology, 95(8), e1060-e1070.

Rossi, A. (2008). Imaging of acute disseminated encephalomyelitis. Neuroimaging clinics of North America, 18(1), 149-161.

Scullen, T., Keen, J., Mathkour, M., Dumont, A. S., \& Kahn, L. (2020). Coronavirus 2019 (COVID-19)-associated encephalopathies and cerebrovascular disease: the New Orleans experience. World neurosurgery, 141, e437-e446.

Sepehrinezhad, A., Shahbazi, A., \& Negah, S. S. (2020). COVID-19 virus may have neuroinvasive potential and cause neurological complications: a perspective review. Journal of neurovirology, 1.

Sheraton, M., Deo, N., Kashyap, R., \& Surani, S. (2020). A review of neurological complications of COVID-19. Cureus, 12(5).

Sohal, S., \& Mansur, M. (2020). COVID-19 presenting with seizures. IDCases, 20, e00782.

Steardo, L., Steardo Jr, L., Zorec, R., \& Verkhratsky, A. (2020). Neuroinfection may contribute to pathophysiology and clinical manifestations of COVID-19. Acta Physiologica (Oxford, England), 229, 10-3.

Tassorelli, C., Mojoli, F., Baldanti, F., Bruno, R., \& Benazzo, M. (2020). COVID-19: what if the brain had a role in causing the deaths? European journal of neurology.

Toljan, K. (2020). Letter to the editor regarding the viewpoint "evidence of the COVID-19 virus targeting the CNS: tissue distribution, host-virus interaction, and proposed neurotropic mechanism". ACS chemical neuroscience, 11(8), 1192-1194.

Werner, C., Scullen, T., Mathkour, M., Zeoli, T., Beighley, A., Kilgore, M. D., \& Keen, J. R. (2020). Neurological impact of coronavirus disease of 2019: practical considerations for the neuroscience community. World neurosurgery, 139, 344-354.

Wong, P. F., Craik, S., Newman, P., Makan, A., Srinivasan, K., Crawford, E., \& Ahmad, N. (2020). Lessons of the month 1: a case of rhombencephalitis as a rare complication of acute COVID-19 infection. Clinical Medicine, 20(3), 293-294. 
Research, Society and Development, v. 10, n. 12, e464101220764, 2021

(CC BY 4.0) | ISSN 2525-3409 | DOI: http://dx.doi.org/10.33448/rsd-v10i12.20764

Xu, J., Zhong, S., Liu, J., Li, L., Li, Y., Wu, X., \& Jiang, Y. (2005). Detection of severe acute respiratory syndrome coronavirus in the brain: potential role of the chemokine mig in pathogenesis. Clinical infectious diseases, 41(8), 1089-1096.

Xu, Z., Shi, L., Wang, Y., Zhang, J., Huang, L., Zhang, C., \& Wang, F. S. (2020). Pathological findings of COVID-19 associated with acute respiratory distress syndrome. The Lancet respiratory medicine, 8(4), 420-422.

Ye, M., Ren, Y., \& Lv, T. (2020). Encephalitis as a clinical manifestation of COVID-19. Brain, behavior, and immunity, 88, 945-946.

Zayet, S., Royer, P. Y., Toko-Tchiundzie, L., Gendrin, V., \& Klopfenstein, T. (2020). Encephalopathy in patients with COVID-19:'Causality or coincidence?'. Journal of medical virology, 1-3.

Zubair, A. S., McAlpine, L. S., Gardin, T., Farhadian, S., Kuruvilla, D. E., \& Spudich, S. (2020). Neuropathogenesis and neurologic manifestations of the coronaviruses in the age of coronavirus disease 2019: a review. JAMA neurology, 77(8), 1018-1027. 